\title{
Overexpression of acid-sensing ion channel 1a (ASIC1a) promotes breast cancer cell proliferation, migration and invasion
}

\author{
Chao Yang ${ }^{1 \#}$, Zhen Zhu $^{2 \#}$, Xueyan Ouyang ${ }^{1}$, Ruihua Yu ${ }^{1}$, Jiawei Wang ${ }^{2}$, Gang Ding ${ }^{3}$, Feng Jiang ${ }^{1 \wedge}$ \\ ${ }^{1}$ Translational Institute for Cancer Pain, Xinhua Hospital Affiliated to Shanghai Jiao Tong University School of Medicine, Chongming Branch, \\ Shanghai, China; ${ }^{2}$ School of Life Sciences, Shanghai University, Shanghai, China; ${ }^{3}$ Department of Oncology, Shanghai International Medical Center, \\ Shanghai, China \\ Contributions: (I) Conception and design: F Jiang, G Ding; (II) Administrative support: J Wang, R Yu, Z Zhu; (III) Provision of study materials or \\ patients: C Yang, X Ouyang, Z Zhu; (IV) Collection and assembly of data: C Yang, Z Zhu; (V) Data analysis and interpretation: C Yang, Z Zhu; (VI) \\ Manuscript writing: All authors; (VII) Final approval of manuscript: All authors. \\ "These authors contributed equally to this work. \\ Correspondence to: Feng Jiang, PhD. Associate professor, Translational Institute for Cancer Pain, Xinhua Hospital Affiliated to Shanghai Jiao Tong \\ University School of Medicine, Chongming Branch, Shanghai 202150, China. Email: fengjiang@xinhuamed.com.cn; Gang Ding, PhD. Chief \\ Physician, Department of Oncology, Shanghai International Medical Center, Shanghai 201318, China. Email: ddinggang0608@163.com.
}

Background: The microenvironment of various tumor tissues is acidic. Acid-sensing ion channels (ASICs) are a class of ligand-gated ion channels which are sensitive to extracellular protons and are often highly expressed in tumor tissues. Breast cancer, whose extracellular microenvironment is thought to be acidic, is the most common cancer type among females in the world.

Methods: Thirty breast cancer tissues and adjacent normal tissues of patients were collected from 2009 to 2015 at the Xinhua hospital affiliated to Shanghai Jiao Tong University School of Medicine. The expression of acid-sensing ion channel 1a (ASIC1a), a subtype of ASICs family, was detected by immunohistochemistry in breast cancer tissues, and the effect of ASIC1a on the physiological activity of tumor cells was analyzed in vitro and in vivo experiments.

Results: In this study, it was found that ASIC1a is highly expressed in the tissues of breast cancer patients. In vitro experiments revealed that down-regulation of ASIC1a by its antagonist PcTx-1 or ASIC1a siRNA could significantly weaken the migration, proliferation and invasion of tumor cells. In vivo studies, downregulation or inhibition of the ASIC1a could inhibit breast tumor growth.

Conclusions: The high expression of ASIC1a might be related to the enhanced biological activity of breast cancer cells. Whether ASIC1a is a potential therapeutic target for some types of breast cancer deserves further study.

Keywords: Acid-sensing ion channel 1a (ASIC1a); breast cancer; psalmotoxin 1 (PcTx-1); siRNA

Submitted May 18, 2020. Accepted for publication Oct 26, 2020.

doi: $10.21037 /$ tcr-20-2115

View this article at: http://dx.doi.org/10.21037/tcr-20-2115

\section{Introduction}

Breast cancer is the most common cancer type among females in the world and is considered as the second cancer type frequently occurring worldwide among newly- diagnosed cancers (1-4). In China, the number of patients with breast cancer has accounted for $12.2 \%$ of all breast cancer patients globally (5). Therefore, breast cancer has been thought to be the $6^{\text {th }}$ leading cause of death for females

^ ORCID: 0000-0002-8071-8279 
in China (6), bringing about a serious threat to the life and health of women.

The diagnosis and treatment level of breast cancer has been improving year by year, and the 5 -year survival rate has reached $90 \%$ (7). The systemic treatment of breast cancer has initially formed a mature system including chemotherapy, endocrine therapy, targeted therapy and immunotherapy. Chemotherapy is still the main method of systemic treatment of breast cancer, but chemotherapy has serious adverse reactions and is prone to drug resistance. Existing data have shown that several intracellular proteins are abnormally expressed in breast cancer cells and have been developed as therapeutic targets for the treatment of breast cancer, such as human epidermal growth factor receptor-2 (HER2), cyclooxygenase-2 (COX-2), epidermal growth factor receptor (EGFR), vascular endothelial growth factor (VEGF) (8-12). Therefore, drugs targeting for these proteins have been designed and found to be specific to certain types of breast cancer, especially in patients with positive HER2 breast cancer, targeted therapy has achieved good effect. Immunotherapy has also made progress in the treatment of triple negative breast cancer, the phase III clinical study IMpassion 130 demonstrated that immunotherapy was effective in first-line treatment of triple negative breast cancer (13), but its effect is poor compared with lymphoma, lung cancer, skin cancer and so on. But the traditional chemotherapy was still excelled in efficiency and median survival than the targeted drugs. Therefore, the molecular mechanisms of breast cancer regulated by these proteins remain to be uncovered.

Acid-sensing ion channels (ASICs) are a class of ligandgated ion channels that are widely distributed in muscle, joint and soft tissue as well as in the peripheral and central nervous systems $(14,15)$. Recent studies have revealed that ASICs are widely involved in important physiological and pathological processes such as development, sensation, learning and memory, nerve damage and pain (16). Since tumor cells had a high rate of glucose metabolism and limited blood supply, an acidic microenvironment was found in tumor and around tumor tissues $(17,18)$. As an acid-sensing channel, ASIC1a might play a key role in the development of tumors. It has been reported that ASIC1a mRNA is highly expressed in both in normal and lowgrade glioma cells (19). Native psalmotoxin 1 (PcTx-1) is a 40 -amino acid toxin originally isolated from the venom of the South American tarantula Psalmopoeus cambridgei. PcTx1 potently (IC50 $=0.9 \mathrm{nM}$ ) and specifically blocks the acidsensing ion channel 1a (ASIC1a) and is able to discriminate between the two splice variant subtypes and not block the ASIC1b channel (20). Blocking ASIC1a with PcTX1 could specifically reduce the migration and proliferation of D54-MG glioma cells (21). Down-regulation of ASIC1a and epithelial sodium channels could inhibit the invasion and metastasis of glioma cells (22). Silencing of ASIC1a with miRNAs reduced the proliferation and migration of hepatoma cells (23). Gupta et al.'s study showed that ASIC1 activates the ROS-AKT-NF- $\mathrm{KB}$ signaling pathway by increasing calcium influx under acidic conditions, which regulates proliferation, migration and invasion of tumor cells. Inhibition of ASIC1 by amiloride were found to inhibit the proliferation of tumor cells and prolong the survival time of rat with breast cancer (24). However, which subtype of ASICs is involved in breast cancer has not yet been fully discovered. In the current study, we aimed to study the involvement and underlying mechanisms of ASIC1a in breast cancer. We present the following article in accordance with the MDAR reporting checklist (available at http://dx.doi.org/10.21037/tcr-20-2115).

\section{Methods}

\section{Cell lines}

Breast cancer cell lines MCF-7 and MDA-MB-231were obtained from Cell Bank of Type Culture Collection of Chinese Academy of Sciences (Shanghai, China) and cultured in Dulbecco's Modified Eagle's Medium (DMEM, Gibco, USA) supplemented with 10\% FBS (HyClone, USA). The medium was changed every 2 days. Cells were passaged and digested with $0.25 \%$ trypsin for cell fusion as a single layer.

\section{Reagents}

The antibodies used: anti- $\beta$-actin, anti-ASIC1a were from Abcam (Cambridge, UK), other antibodies were purchased from Beyotime (Shanghai, China).

PcTx-1: ASIC1a special inhibitor was produced by Peptide (Peptide Institute, Inc., Japan). All other chemicals were purchased from Sangon Biotech (Shanghai, China) unless otherwise stated.

\section{Cancer tissues}

Thirty breast cancer tissues and adjacent normal tissues of patients were collected from 2009 to 2015 at the Xinhua 
Hospital affiliated to Shanghai Jiao Tong University School of Medicine. All the tumor tissue donors knew about the experiment and signed the informed consent. The stage of tumor was determined according to the TNM system of the American Joint Committee and Union for International Cancer Control on Cancer (25).

The research respects and fully protects the patients' right to know and privacy. Before the sample collection, each patient and guardian should be fully informed of the research content, obligations and responsibilities, adverse reactions and the process of joining and withdrawing, and the informed consent for clinical sample scientific research should be signed. The inclusion of patients follows the following principles:

Enrollment criteria: (I) simple invasive breast cancer patients, no other systemic diseases; (II) 20-80 years old; (III) good awareness, able to communicate with researchers; (IV) preoperative chemotherapy, radiotherapy and other antitumor therapy were not performed.

Exclusion criteria: (I) patients with concomitant tumor; (II) patients with other systemic diseases; (III) tumor brain metastases or severe cognitive impairment, such as psychiatric patients; (IV) other items that do not meet the inclusion criteria. Rejection criteria: (I) serious complications or other systemic diseases occurred during follow-up; (II) incomplete information due to death or loss during follow-up.

\section{Immunobistochemistry}

Formalin-fixed, paraffin-embedded tumor tissue sections $(6 \mu \mathrm{m})$ were deparaffinized in xylene and rehydrated with gradient concentrations of ethanol. Endogenous peroxidase activity was blocked ( $3 \% \mathrm{H}_{2} \mathrm{O}_{2}$ in $\mathrm{PBS}$ buffer), antigens were retrieved by microwaving $(350 \mathrm{~W})$, and nonspecific binding was blocked by $5 \%$ bovine serum albumin in PBS buffer. Sections were stained with ASIC1a antibody (diluted in 1:500; ab76203, Abcam) and visualized with secondary antibody (diluted in 1:1,000, Beyotime, A0208). Then, these tumor slides were incubated with 3,3'-diaminobenzidine chromogen (P0203, Beyotime), counterstained in hematoxylin (C0107, Beyotime). The staining area and integral optical density (IOD) of immunohistochemistry were assayed by Image pro plus 6.0 (Media Cybernetics, Inc., USA).

\section{MTT assay}

Cells were transfected with ASIC1a-siRNA or GFP-
siRNA by RNAi-Mate (G04002, GenePharma, Shanghai, China) over night. Cells treated with PcTx-1 were cultured in DMEM with a final concentration of 100 or $200 \mathrm{ng}$ of the drug and plated in 96-well plates at a density of 3,000 cells/well. The cell growth was determined by 3-(4,5-methylthiazol-2-yl)-2,5 diphenyltetrazolium bromide (MTT) colorimetric growth assay. Every 24 h, the cell growth was determined by adding MTT solution (20 $\mu \mathrm{L} /$ well) for $3 \mathrm{~h}$. Cellular MTT was solubilized with acidic isopropanol and optical density was measured at $570 \mathrm{~nm}$. The doubling time was calculated for the exponential growth phase. All experiments were performed 3 times independently. The viability of the cells was assessed from three replicates in three independent experiments by the MTT.

\section{Western blot assay}

Breast cancer cell lines were lysed with cell lysis buffer (50 mM, pH7.5) containing: $150 \mathrm{mM} \mathrm{NaCl}, 1 \%$ Triton $\mathrm{X}-100,2 \mathrm{mM}$ sodium pyrophosphate, $25 \mathrm{mM}$ $\beta$-glycerophosphate, $1 \mathrm{mM}$ EDTA, $1 \mathrm{mM} \mathrm{Na} \mathrm{VO}_{4}$, $0.5 \mu \mathrm{g} / \mathrm{mL}$ leupeptin (Beyotime, P0013). The concentration of protein samples were quantified using Enhanced BCA Protein Assay Kit (P0009B, Beyotime), then the same amount of protein were resolved by SDS-PAGE (12\%), proteins were transferred to PVDF membranes (Roche Incorp., Germany), and these PVDF membranes were incubated with rabbit polyclonal anti-ASIC1a (ab76203, Abcam) or mouse monoclonal anti-actin (A0208, Beyotime) overnight at $4{ }^{\circ} \mathrm{C}$. The protein blots were visualized using secondary HRP-conjugated anti-rabbit or anti-mouse antibodies and BeyoECL Plus (P0018, Beyotime).

\section{Real-time PCR}

Total RNA was extracted using RNA Isolater Total RNA Extraction Reagent (R401-01, Vazyme, Nanjing, China). Quantitative real-time PCR (qPCR) was performed using HiScript II One Step qRT-PCR SYBR Green Kit (R221, Vazyme) on CFX96 real-time PCR system (Bio-Rad, USA). GAPDH was used as an internal control. All samples were normalized to internal controls.

\section{Scratch assay}

The cells were treated as in MTT assay but plated in 6 -well plates at a density of $5 \times 10^{5}$ cells/well and cultured 
overnight, then used a yellow pipette tip to make a straight scratch among cell layer, and washed the cells three times with PBS (pH7.4). The cells were then incubated for $24 \mathrm{~h}$ in DMEM supplemented with $1 \%$ FBS. Scratches were monitored every $24 \mathrm{~h}$ by a microscope camera system. The area of scratches was measured by Image pro plus 6.0.

\section{Tumor invasion assay}

Twenty-five $\mu g$ Matrigel basement membrane (356237, Corning, USA) was paved in the up chamber of the $6.5 \mathrm{~mm}$ transwell chamber with $8.0 \mu \mathrm{m}$-pore Polycarbonate Membrane Insert (3422, Corning Costa, USA). Six hundred $\mu \mathrm{L}$ serum-free medium to transwell upper and lower chambers for $12 \mathrm{~h}$. Seven hundred $\mu \mathrm{L} 10 \%$ serum medium containing the 100 or $200 \mathrm{ng}$ of PcTx-1 was then added into the lower chamber. The cells were then plated in the up chamber with serum-free medium at a density of $1 \times 10^{4}$ cells/well and incubated in a cell incubator for $24 \mathrm{~h}$. The cells which did not cross the basement membrane in the up chamber were removed from the membrane, stained with $0.1 \%$ crystal violet solution for $30 \mathrm{~min}$, and count the number of invasive cells.

\section{Animals}

Nude mice (BALB/C, female, about 3 weeks of age) were employed and raised under SPF conditions. All in vivo experiments were performed according to our institutions' guidelines for the use of laboratory animals.

For orthotopic implantation, $5 \times 10^{7} \mathrm{MCF}-7$ cells or MDA-MB-231 cells suspended in $100 \mu \mathrm{L}$ PBS were injected into the mammary fat pad of nude mice. Mice were randomly assigned into three independent groups (4 mice in each group), control group, Pc Tx-1 group (10 ng/kg, tail vein injection, every day for 7 days), ASIC1a siRNA group (MCF-7 cells or MDA-MB-231 cells were transfected with ASIC1a siRNA, and then injected into the mammary fat pad of nude mice). On day 21, animals were euthanized and tumors were excised and weighted.

\section{Statistical analyses}

The R Statistical Software Package 3.1.3 was used to analyze all the data. The data was report as mean \pm SD to display variability. Independent Student's $t$-test was used to analyze differences. The relationship between ASIC1a mRNA in control group and testing group were analyzed by the Mann-Whitney test. The significant difference was determined by $\mathrm{P}<0.05$.

\section{Ethical approval/statement}

The study was conducted in accordance with the Declaration of Helsinki (as revised in 2013). The study was approved by the ethnical committee of Shanghai International Medical Center (SIMC-EC-2019-03) and informed consent was taken from all the patients. Animals experiments were performed under a project license (NO.: 2019YA03) granted by Academic Management Committee of Xinhua Hospital Affiliated with Shanghai Jiao Tong University School of Medicine Chongming Branch, in compliance with the institutions' guidelines for the use of laboratory animals.

\section{Results}

\section{Expression of ASIC1a in breast tumor tissues}

The expression of ASIC 1 a in 30 pairs of tumor tissues and adjacent normal tissues was detected by immunohistochemical staining. The result showed that the expression of ASIC1a in tumor tissues was significantly up-regulated (Figure 1A), which was approximately 2-fold higher than that of normal tissues $(\mathrm{P}<0.01$, Figure $1 B)$.

\section{Down-regulation of ASIC1a inbibit the proliferation, migration and invasion of breast cancer cells}

ASIC1a-specific siRNA was designed and used to downregulate the expression of ASIC1a in breast cancer cell lines MDA-MB-231 and MCF-7 in vitro. As showed in Figure 2, the mRNA and protein expression of ASIC1a showed no significant difference between GFP-siRNA transfected cells and control cells. However, ASIC1a mRNA and protein expression reduced more than $50 \%$ in ASIC1a-siRNA transfected cells.

As shown in Figure $2 C$, compared with GFP-siRNA transfected cells and control cells, the proliferation of ASIC1a-siRNA transfected cells was significantly reduced with a proliferation rate of $59.97 \% \pm 3.46 \%$ in MDAMB-231 cell lines $(\mathrm{P}<0.01)$. and $75.55 \% \pm 6.39 \%$ in $\mathrm{MCF}-7$ cell lines $(\mathrm{P}<0.01)$.

The mobility of the two breast cancer cell lines was significantly inhibited by ASIC1a-siRNA in MDA-MB-231 cells, the distance of migration was $147.90 \pm 15.93 \mu \mathrm{m}$ in 
A
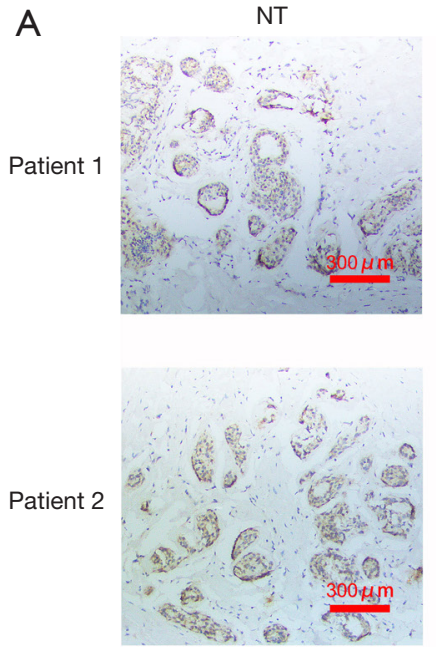

$\mathrm{T}$
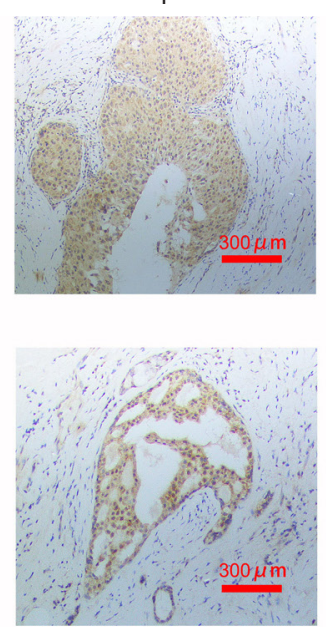

B

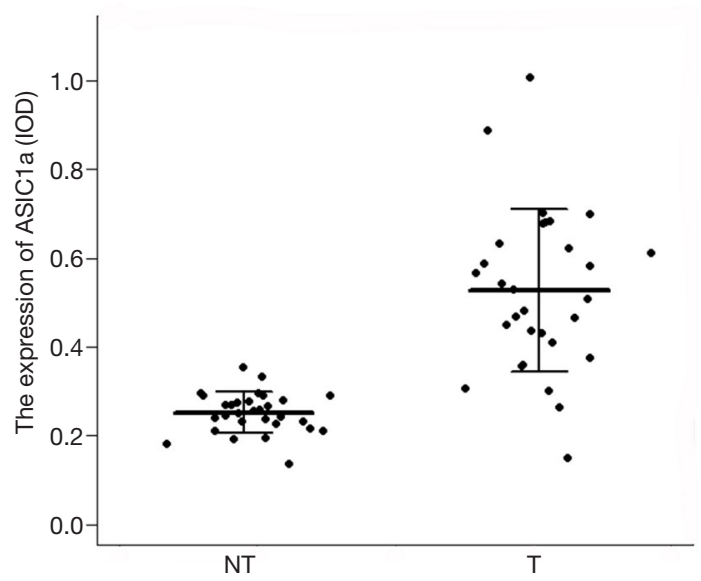

Figure 1 Expression of ASIC1a in breast tumor tissues. (A) Expression of ASIC1a in adjacent normal tissues (NT) and breast cancer tissues (T); (B) the relative expression of ASIC1a in 30 breast cancer tissues were normalized by the expression of ASIC1a expression in adjacent normal tissues. ASIC1a, acid-sensing ion channel 1a; IOD, integral optical density.

ASIC1a-siRNA transfected group, $421.29 \pm 11.50 \mu \mathrm{m}$ in GFP-siRNA transfected group and $439.87 \pm 8.84 \mu \mathrm{m}$ in control group (Figure $3 A$ ). In MCF-7 cells, the migration distance was $159.95 \pm 33.22 \mu \mathrm{m}$ in control group was $136.67 \pm 12.87 \mu \mathrm{m}$ in GFP-siRNA transfected group and $66.67 \pm 10.30 \mu \mathrm{m}$ in ASIC1a-siRNA transfected group (Figure $3 B)$. These results indicated that the migration ability of the breast cancer cells was decreased due to the downregulation of ASIC1a in MDA-MA-231 and MCF-7 cells.

Down-regulation of ASIC1a in MDA-MB-231 and MCF-7 cells was also found to inhibit the invasion of breast cancer cells. After transfection of ASIC1a-siRNA in MDAMB-231 and MCF-7 cells, the invasive ability were reduced significantly (Figure $3 A, B$ ). The number of invading cells in MDA-MB-231 was $139.16 \pm 7.39$ after transfection with ASIC1a siRNA, which was significantly less than that in the control $(340.00 \pm 11.31)$ and GFP-siRNA $(337.67 \pm 11.80)$ transfection cells (Figure 3C). Similarly, in MCF-7 cells, the number of invading cells was $91.50 \pm 43.64$ in ASIC1asiRNA transfected cells, significantly less than that in control group $(153.33 \pm 37.12)$ and GFP-siRNA transfected group $(132.67 \pm 30.38)$ (Figure $3 D)$.

\section{PcTx-1 inhibit the proliferation, migration and invasion of breast cancer cells}

PcTx-1 was purified from the venom of Psalmopoeus cambridgei (26). Different with the findings mentioned above, PcTx-1 did not modulate the mRNA level and expression of AISC1a (Figure 4). To further investigate the effect of PcTx-1 on cell functions, we used different concentrations of PcTx-1 to inhibit the activity of ASIC1a.

The MTT assay showed that the proliferation rate of MDA-MB-231 and MCF-7 was decreased with increase of PcTx-1 concentration (Figure 4C,D). In both cell lines, after the treatment of PcTx-1 for $72 \mathrm{~h}$, the average decrease ratio of $100 \mathrm{ng}$ PcTx-1 treated cells was approximately $20 \%$, while the average decrease ratio of $200 \mathrm{ng} \mathrm{PcTx}-1$ treated cells was $35-45 \%$.

The results also showed that ASIC1a inhibitor PcTx-1 could inhibit the migration of MDA-MA-231 and MCF-7 cells (Figure 5A,B). In MDA-MA-231 cells, 100 ng PcTx-1 reduced the migration distance of cells by $162.06 \mu \mathrm{m}$, while $200 \mathrm{ng}$ PcTx-1 reduced the migration distance of cells by $323.74 \mu \mathrm{m}$ (Figure 5A). Similarly, in MCF-7 cells, $100 \mathrm{ng}$ PcTx-1 could reduce the migration distance of cells by $47.32 \mu \mathrm{m}$, while $200 \mathrm{ng} \mathrm{PcTx}-1$ could reduce the migration distance of cells by $106.26 \mu \mathrm{m}$ (Figure $5 B$ ).

The invasion of MDA-MB-231 and MCF-7 cells were found to be inhibited by PcTX-1 (Figure 5C,D). In control MDA-MB-231 cells, the number of invading cells was $378.83 \pm 16.04$, but that in $100 \mathrm{ng} \mathrm{PcTx}-1$ treated group was $186.83 \pm 11.72$ and $92.17 \pm 8.52$ in $200 \mathrm{ng} \mathrm{PcTx}-1$ treated group (Figure 5C). Similarity in MCF-7 cells, the number of invading cells in control group was $179.83 \pm 47.35$, while those in 100 or $200 \mathrm{ng}$ PcTx-1 treated cells were 
A

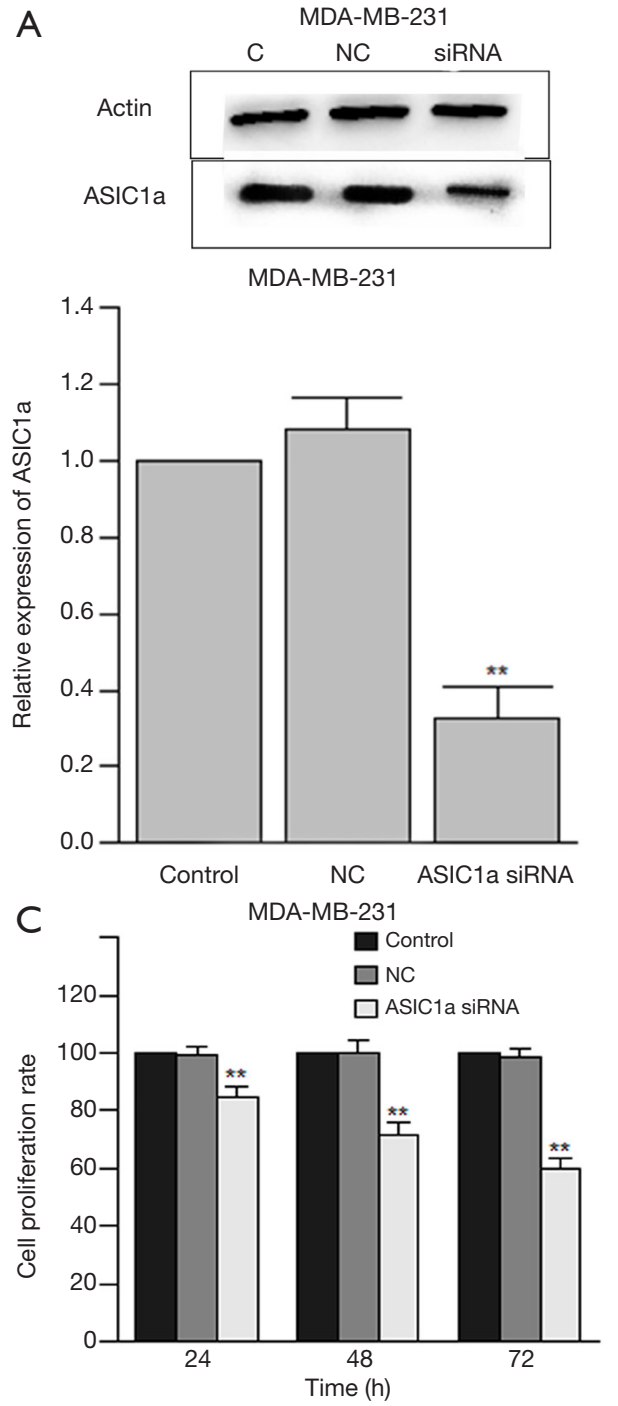

B
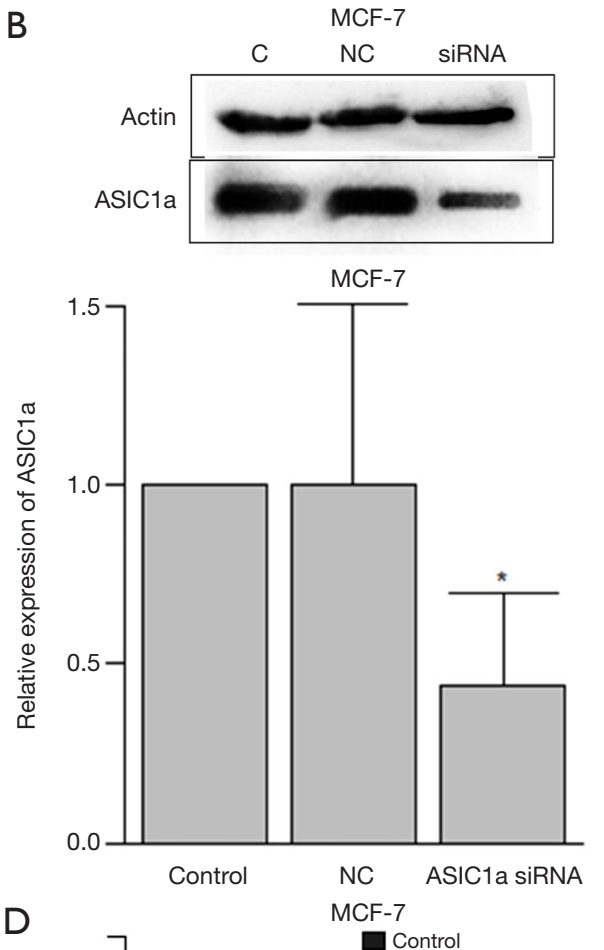

D

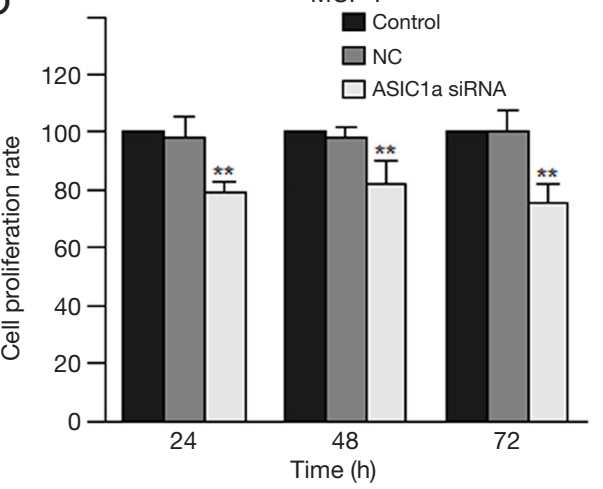

Figure 2 Down-regulation of ASIC1a inhibited the proliferation of breast cancer cells. (A) Western blot of ASIC1a in MDA-MB-231 control cells, GFP-siRNA cells and ASIC1a-siRNA cells; (B) relative expression of ASIC1a in MCF-7 control cells, GFP-siRNA cells and ASIC1a-siRNA cells; MTT assays showed the time-dependent proliferation ability of MDA-MB-231 (C) and MCF-7 (D) cells for control, GFP-siRNA and ASIC1a-siRNA treated groups ( $\mathrm{n}=6$; * $\mathrm{P}<0.05 ;$ **, $\mathrm{P}<0.01, t$-test). ASIC1a, acid-sensing ion channel 1a.

$103.00 \pm 11.58$ or $78.67 \pm 22.22$ (Figure $5 D$ ), respectively.

\section{Down-regulation or inbibition of ASIC1a inbibited liver cancer cells growth in vivo}

We examined whether ASIC1a could affect the growth of breast cancer cells in vivo. MDA-MB-231 cell lines and MCF-7 cell lines were used to construct orthotopic tumor model. The results showed that in the orthotopic tumor model of breast cancer, the tumors in ASIC1a siRNA transfected group and $\mathrm{PcTx}-1$ treated group were significantly smaller than that in the control groups (Figure 6, $\mathrm{P}<0.05$ ). Our data showed that inhibiting or decreasing the expression of ASIC1a inhibits the growth of breast cancer cells in vivo.

\section{Discussion}

Some studies have shown that the acidification of tumor tissue promoted the accumulation of $\mathrm{H}^{+}$in the cell microenvironment, and $\mathrm{H}^{+}$can compete with $\mathrm{Ca}^{2+}$ to bind to the ASIC1a to depolarize the cell and triggered the internal 
A
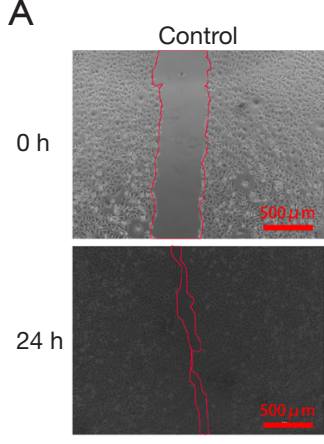

B

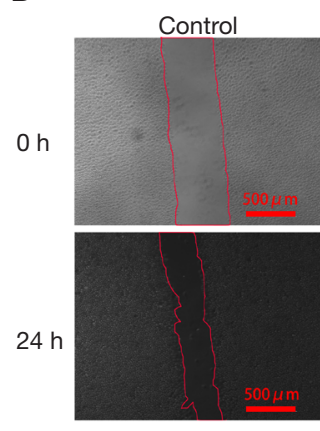

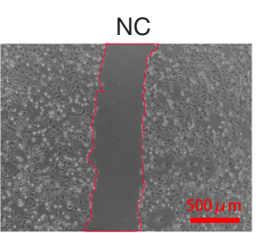
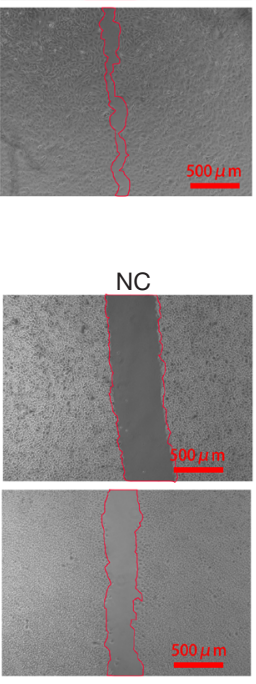

MDA-MB-231
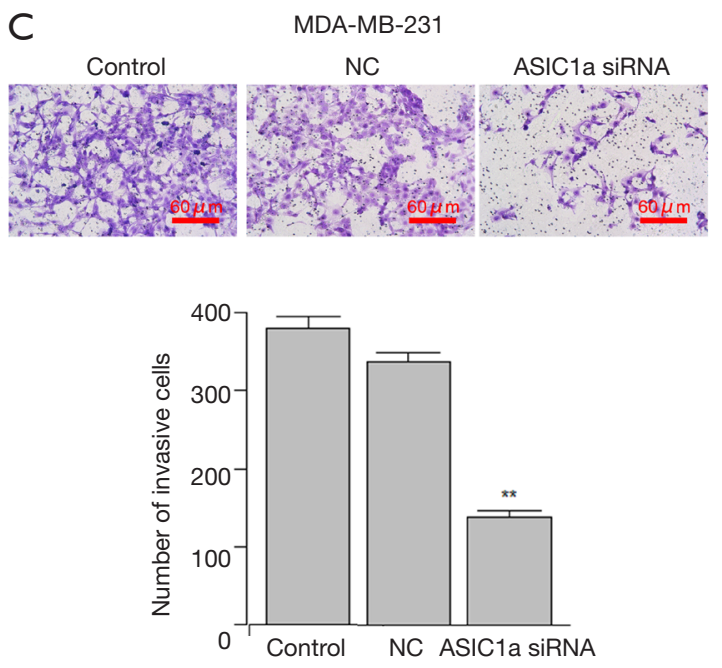

MDA-MB-231

ASIC1a siRNA

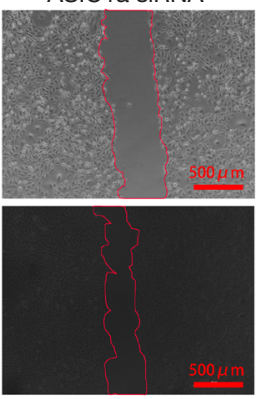

MCF-7
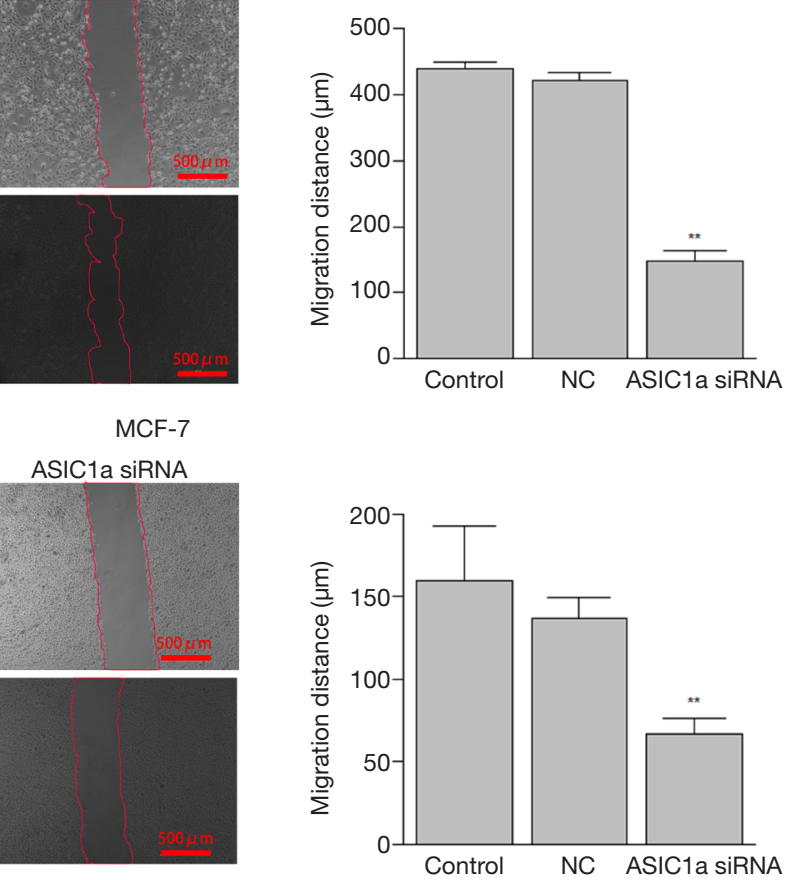

D

MCF-7

$\mathrm{NC}$ ASIC1a siRNA
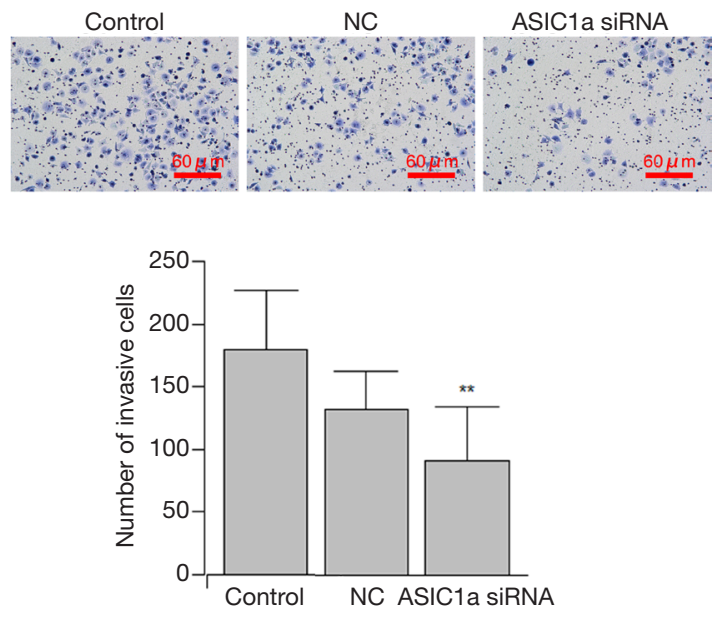

Figure 3 Down-regulation of ASIC1a inhibited the migration and invasion of breast cancer cells. (A) Scratch results of MDA-MB-231 control group, GFP-siRNA group and ASIC1a-siRNA group $24 \mathrm{~h}$ after transfection; the average migration distance of MDA-MB-231 was calculated from the reduced area/image height ( $\mathrm{n}=6$; ** $\mathrm{P}<0.01$, t-test); (B) scratch test results of MCF-7 control group, GFP-siRNA group and ASIC1a-siRNA group $24 \mathrm{~h}$ after transfection; the average migration distance of MCF-7 was calculated from the reduced area/image height ( $\mathrm{n}=6$; **, $\mathrm{P}<0.01$, t-test). (C,D) Representative results of invasion ability of MDA-MB-231 (C) and MCF-7 (D) control, GFP-siRNA and ASIC1a-siRNA transfected cells and the number of invasive cells in MDA-MB-231 and MCF-7 control, GFP-siRNA and ASIC1asiRNA transfected cells was calculated $(\mathrm{n}=6$; **, $\mathrm{P}<0.01, t$-test). ASIC1a, acid-sensing ion channel 1a. 
A
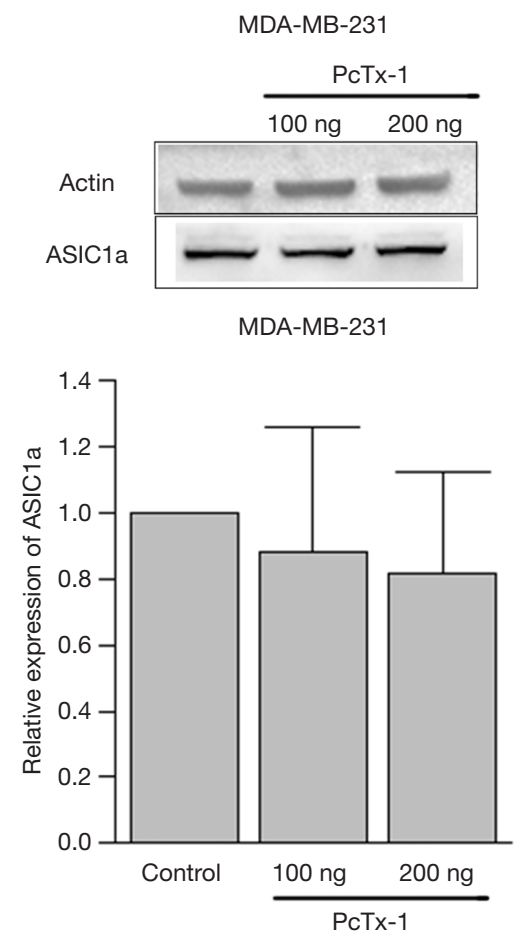

C

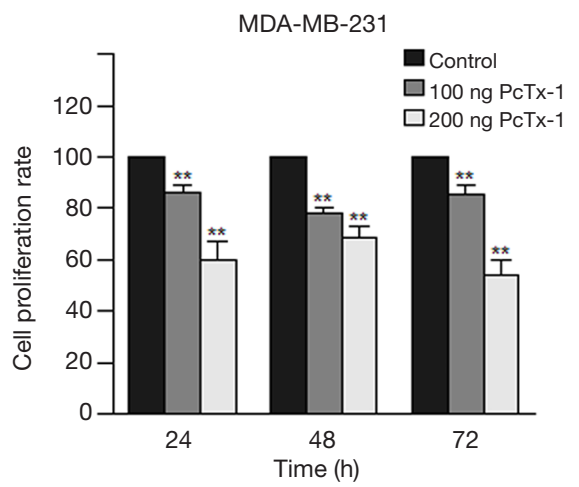

B

B MCF-7
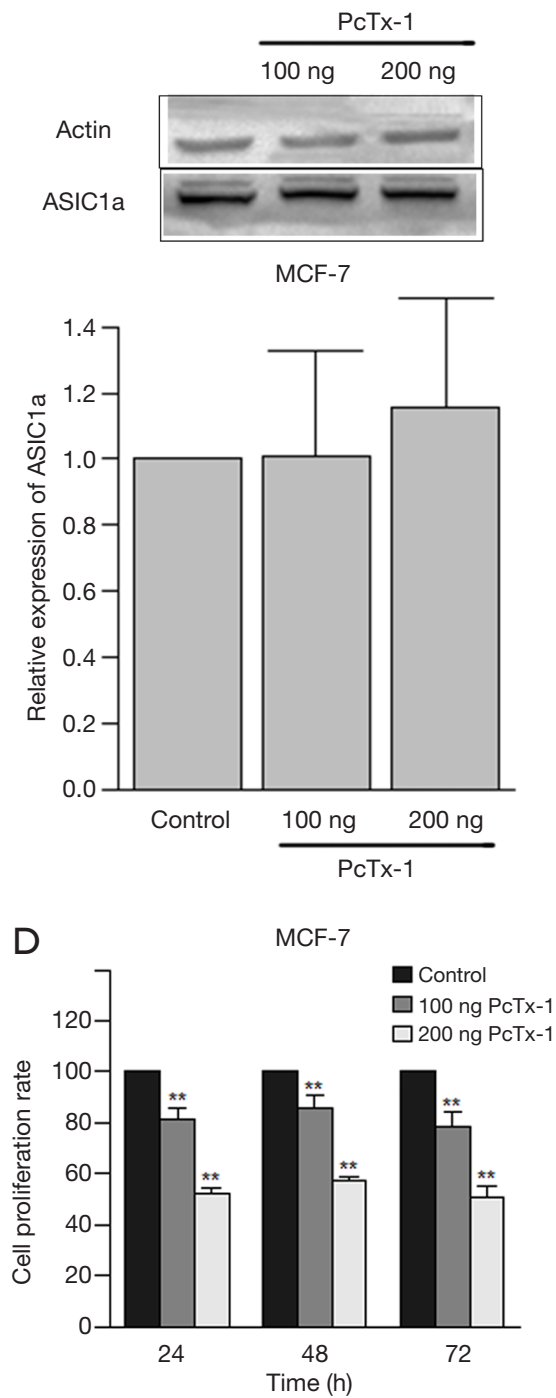

Figure 4 PcTx-1 inhibited the proliferation of breast cancer cells. (A,B) The expression of ASIC1a in MDA-MB-231 (A) or MCF-7 (B) control cells and cells treated with 100 or $200 \mathrm{ng} \mathrm{PcTx}-1$ (up panel) and the relative expression of ASIC1a in control cells, $100 \mathrm{ng}$ and 200 ng PcTx-1 treated MDA-MB-231 (A) or MCF-7 (B) cells (bottom panel). (C,D) Proliferation ability of MDA-MB-231 (C) and MCF-7 (D) control cells, cells treated with 100 or $200 \mathrm{ng}$ PcTx-1 (n=6; **, P<0.01, t-test). ASIC1a, acid-sensing ion channel 1a; PcTx-1, psalmotoxin 1.

flow of $\mathrm{Ca}^{2+}(27,28)$. It is known that $\mathrm{Ca}^{2+}$ is involved in the ubiquitination and phosphorylation of $\beta$-actin protein (29). $\beta$-catenin/LEF-TCF signaling pathway promotes downstream oncogenes expression (30). Similar in tumor cells, down-regulation of ASIC1a expression could reduce the expression of oncogenes by down-regulating $\beta$-catenin/ LEF-TCF. "Acidosis-ROS-PTEN-AKT-NF- $\kappa B$ " axis is an important pathway for intracellular acid environment which is associated with the invasive ability of breast cancer cells by. First, acidic microenvironment could promote reactive oxygen species (ROS) accumulation. Second, $\mathrm{AKT}$ and NF- $\mathrm{\kappa B}$ activation are also increased by ROS accumulation. Third, NF- $\mathrm{\kappa B}$ is a key factor responsible for cell invasion (31). Therefore, the activation of AKT and $\mathrm{NF}-\mathrm{\kappa B}$ could promote the invasion of breast cancer cells in acid microenvironment while blocking ASICs can inhibit the effect of acid environment on breast cancer cells (32). In this study, we hypothesized that high expression of ASIC1a 
A

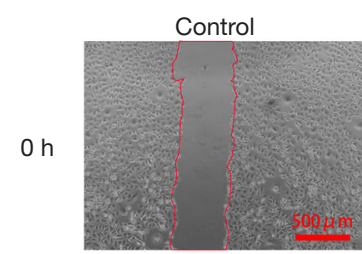

B
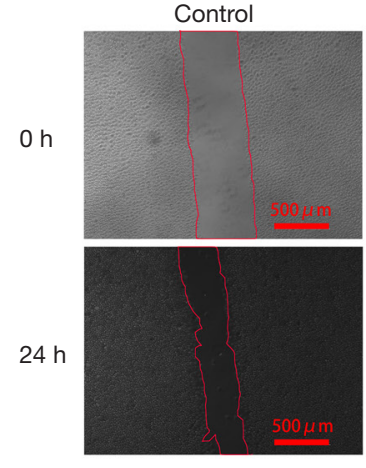

C
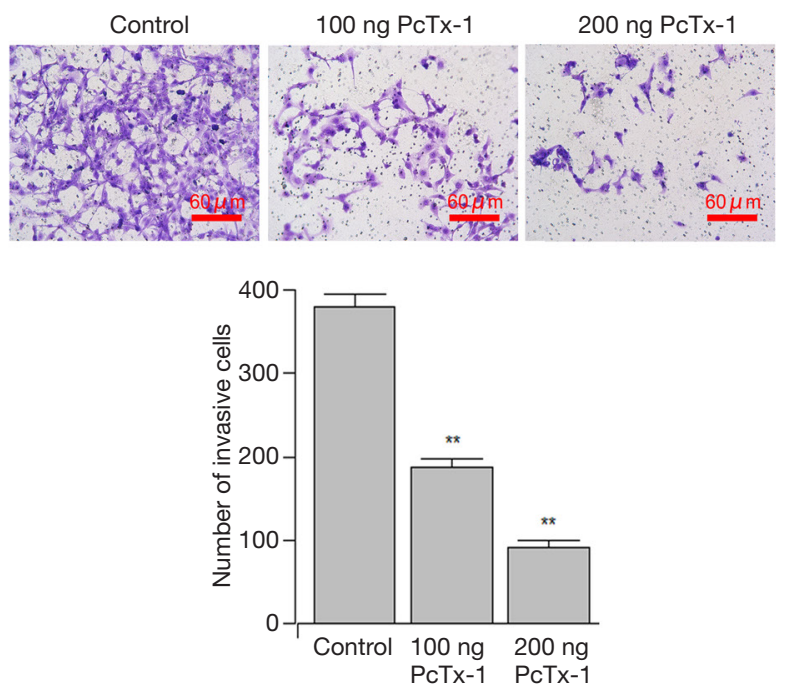
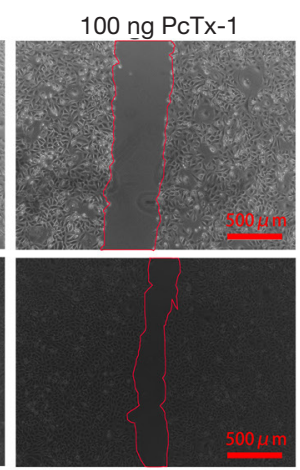

MDA-MB-231

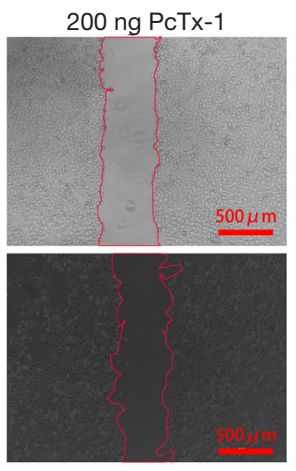

MCF-7 200 ng PcTx-1
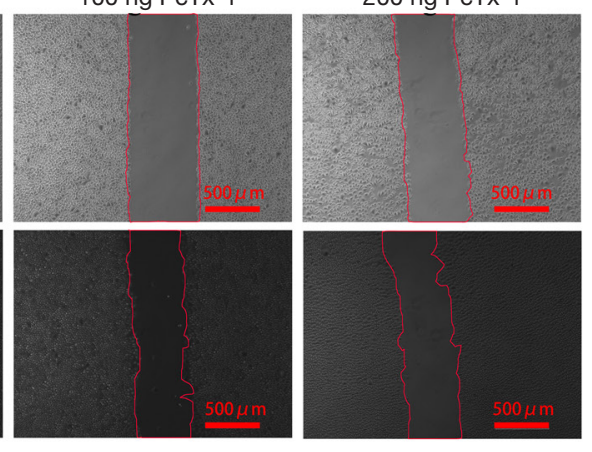

D
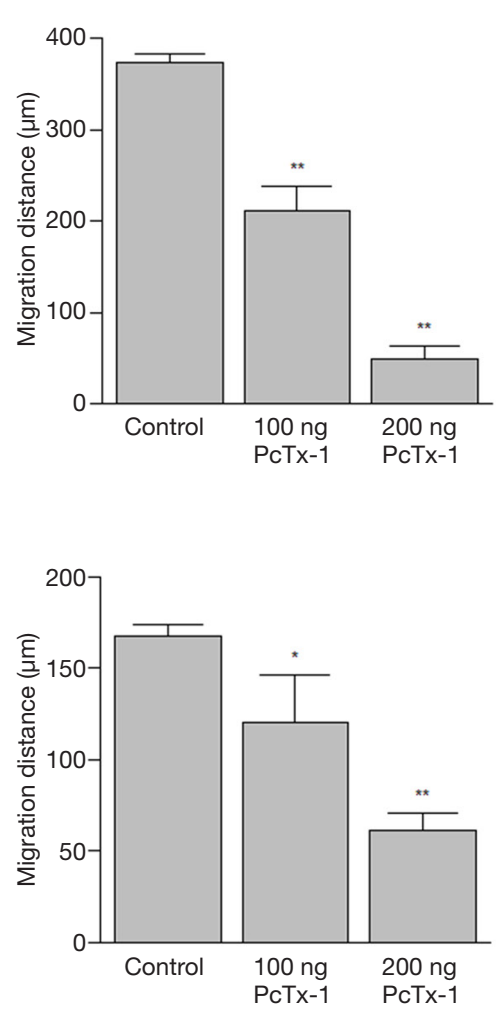

MCF-7
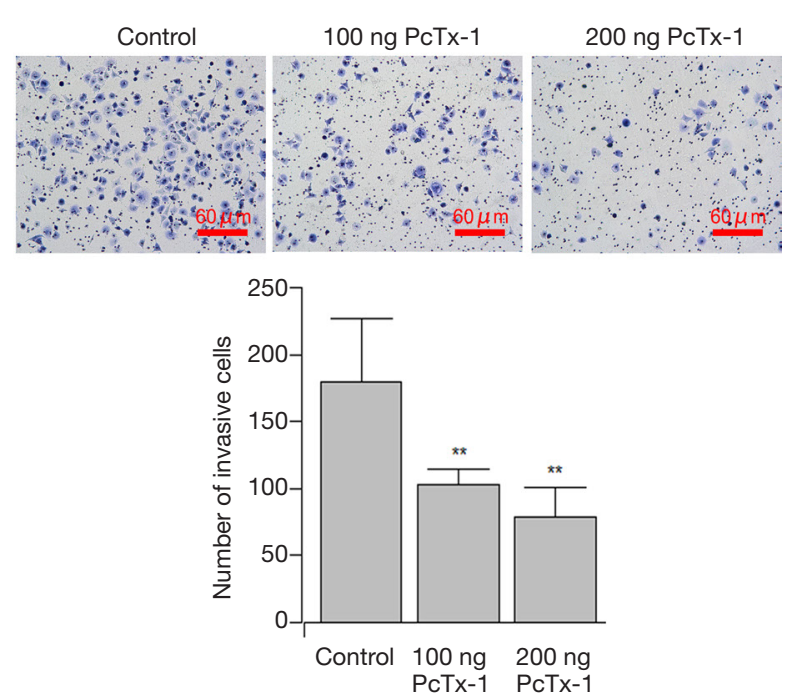

Figure 5 PcTx-1 inhibited the migration and invasion of breast cancer cells. (A,B) Scratch results of MDA-MB-231 (A) and MCF-7 (B) control cells, cells treated with $100 \mathrm{ng}$ or $200 \mathrm{ng}$ PcTx-1. The average migration distances of MDA-MB-231 (A) and MCF-7 (B) cells were calculated from the reduced area/image height ( $\mathrm{n}=6$; **, $\mathrm{P}<0.01$, t-test). (C,D) Representative invasion results of control cells, cells treated with 100 or $200 \mathrm{ng}$ PcTx-1 in MDA-MB-231 (C) and MCF-7 cells (D), and the number of invasive cells in MDA-MB-231 and MCF-7 cells ( $\mathrm{n}=6 ;{ }^{* *}, \mathrm{P}<0.01$, t-test). PcTx-1, psalmotoxin 1. 
A
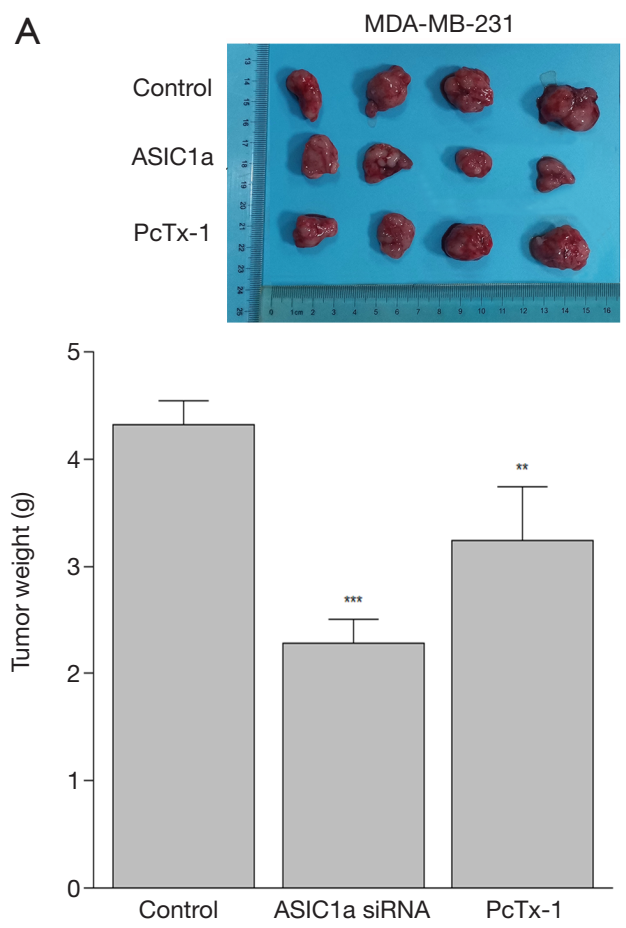

B
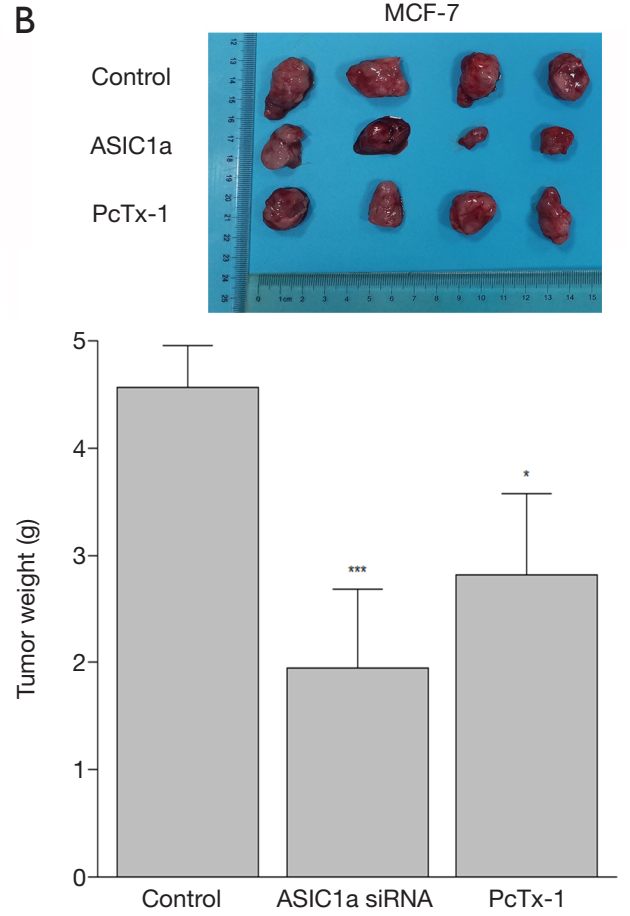

Figure 6 Down-regulation or inhibition of ASIC1a inhibited liver cancer cells growth in vivo. (A) Orthotopic tumor formative ability of ASIC1a siRNA and PcTx-1 treated MDA-MB-231 cell. $\left({ }^{* *}, \mathrm{P}<0.01\right.$; $\left.{ }^{* * *}, \mathrm{P}<0.001\right)$; (B) orthotopic tumor formative ability of ASIC1a siRNA and PcTx-1 treated MCF-7 cell ( ${ }^{*}, \mathrm{P}<0.05$; $\left.{ }^{* * *}, \mathrm{P}<0.001\right)$. ASIC1a, acid-sensing ion channel 1a; $\mathrm{PcTx}-1$, psalmotoxin 1.

maybe could promotes breast cancer cells proliferation, migration, and invasion through $\beta$-catenin/LEF-TCF signaling pathways and "Acidosis-ROS-PTEN-AKT-NF$\kappa B$ ” pathway.

In this research, we found that down-regulation of ASIC1a in MDA-MB-231 and MCF-7 by ASIC1a-siRNA cloud significantly inhibit the proliferation, migration and invasion of MDA-MB-231 and MCF-7 cells. It can be safely estimated that over-expression of AISC1a in breast tumor may promote the development and metastasis of breast tumor. Since it is difficult to down-regulate the expression of ASIC1a by siRNA in clinic, we used a specific inhibitor, PcTx-1 to inhibit the activity of ASIC1a. The results showed that the proliferation, migration and invasion of breast cancer cells cloud be inhibited by PcTx-1 in a concentration-dependent manner, indicating that ASIC1a may be a new drug target for the treatment of breast cancer.

In summary, ASIC1a play an important role in breast cancer development and metastasis. Further study on the mechanisms of ASIC1a in regulating breast cancer development and metastasis may provide new treatment strategies for the treatment of breast cancer. The study also suggested that PcTx-1 is a candidate for developing new drugs targeting ASIC1a to treat breast cancer.

\section{Acknowledgments}

Funding: This study was supported by National Natural Science Foundation of china (81572859), partly by Shanghai Municipal Health Committee (201840003, 20184Y0016, 201740027, 201640201) and Chongming Science and Technology Committee (CKY201803, CKY2019-6).

\section{Footnote}

Reporting Checklist: The authors have completed the MDAR reporting checklist. Available at http://dx.doi.org/10.21037/ tcr-20-2115

Data Sharing Statement: Available at http://dx.doi. org/10.21037/tcr-20-2115

Conflicts of Interest: All authors have completed the ICMJE uniform disclosure form (available at http://dx.doi. 
org/10.21037/tcr-20-2115). Dr. CY reports grants from Shanghai Municipal Health Committee, Chongming Science and Technology Committee, during the conduct of the study. Dr. GD reports grants from National Natural Science Foundation of china and grants from Shanghai Municipal Health Committee during the conduct of the study. Dr. FJ reports grants from Shanghai Municipal Health Committee, Chongming Science and Technology Committee and Shanghai Municipal Health Committee during the conduct of the study. The other authors have no conflicts of interest to declare.

Ethical Statement: The authors are accountable for all aspects of the work in ensuring that questions related to the accuracy or integrity of any part of the work are appropriately investigated and resolved. The study was conducted in accordance with the Declaration of Helsinki (as revised in 2013). The study was approved by the ethnical committee of Shanghai International Medical Center (SIMC-EC-2019-03) and informed consent was taken from all the patients. Animals experiments were performed under a project license (NO.: 2019YA03) granted by Academic Management Committee of Xinhua Hospital Affiliated with Shanghai Jiao Tong University School of Medicine Chongming Branch, in compliance with the institutions' guidelines for the use of laboratory animals.

Open Access Statement: This is an Open Access article distributed in accordance with the Creative Commons Attribution-NonCommercial-NoDerivs 4.0 International License (CC BY-NC-ND 4.0), which permits the noncommercial replication and distribution of the article with the strict proviso that no changes or edits are made and the original work is properly cited (including links to both the formal publication through the relevant DOI and the license). See: https://creativecommons.org/licenses/by-nc-nd/4.0/.

\section{References}

1. Siegel RL, Miller KD, Jemal A. Cancer statistics, 2018. CA Cancer J Clin 2018;68:7-30.

2. Siegel RL, Miller KD, Jemal A. Cancer Statistics, 2017. CA Cancer J Clin 2017;67:7-30.

3. Siegel RL, Miller KD, Jemal A. Cancer statistics, 2016. CA Cancer J Clin 2016;66:7-30.

4. Torre LA, Bray F, Siegel RL, et al. Global cancer statistics, 2012. CA Cancer J Clin 2015;65:87-108.

5. Fan L, Strasser-Weippl K, Li JJ, et al. Breast cancer in
China. Lancet Oncol 2014;15:e279-89.

6. Wang YC, Wei LJ, Liu JT, et al. Comparison of Cancer Incidence between China and the USA. Cancer Biol Med 2012;9:128-32.

7. Allemani C, Matsuda T, Di Carlo V, et al. Global surveillance of trends in cancer survival 2000-14 (CONCORD-3): analysis of individual records for 37 513025 patients diagnosed with one of 18 cancers from 322 population-based registries in 71 countries. Lancet 2018;391:1023-75.

8. Lin NU, Dieras V, Paul D, et al. Multicenter phase II study of lapatinib in patients with brain metastases from HER2-positive breast cancer. Clin Cancer Res 2009;15:1452-9.

9. Ardavanis A, Doufexis D, Kountourakis P, et al. Salvage therapy of pretreated advanced breast cancer with bevacizumab and paclitaxel every two weeks: a retrospective case review study. BMC Cancer 2009;9:338.

10. Green MD, Francis PA, Gebski V, et al. Gefitinib treatment in hormone-resistant and hormone receptor-negative advanced breast cancer. Ann Oncol 2009;20:1813-7.

11. Hegde PS, Rusnak D, Bertiaux M, et al. Delineation of molecular mechanisms of sensitivity to lapatinib in breast cancer cell lines using global gene expression profiles. Mol Cancer Ther 2007;6:1629-40.

12. Wu K, Wang C, D'Amico M, et al. Flavopiridol and trastuzumab synergistically inhibit proliferation of breast cancer cells: association with selective cooperative inhibition of cyclin D1-dependent kinase and Akt signaling pathways. Mol Cancer Ther 2002;1:695-706.

13. Schmid P, Adams S, Rugo HS, et al. Atezolizumab and Nab-Paclitaxel in Advanced Triple-Negative Breast Cancer. N Engl J Med 2018;379:2108-21.

14. Baron A, Lingueglia E. Pharmacology of acid-sensing ion channels - Physiological and therapeutical perspectives. Neuropharmacology 2015;94:19-35.

15. Liu C, Zhu LL, Xu SG, et al. ENaC/DEG in Tumor Development and Progression. J Cancer 2016;7:1888-91.

16. Lin SH, Sun WH, Chen CC. Genetic exploration of the role of acid-sensing ion channels. Neuropharmacology 2015;94:99-118.

17. Gerweck LE, Seetharaman K. Cellular $\mathrm{pH}$ gradient in tumor versus normal tissue: potential exploitation for the treatment of cancer. Cancer Res 1996;56:1194-8.

18. Rofstad EK, Mathiesen B, Kindem K, et al. Acidic extracellular $\mathrm{pH}$ promotes experimental metastasis of human melanoma cells in athymic nude mice. Cancer Res 
2006;66:6699-707.

19. Berdiev BK, Xia J, McLean LA, et al. Acid-sensing ion channels in malignant gliomas. J Biol Chem 2003;278:15023-34.

20. Escoubas P, De Weille JR, Lecoq A, et al. Isolation of a tarantula toxin specific for a class of proton-gated $\mathrm{Na}+$ channels. J Biol Chem 2000;275:25116-21.

21. Rooj AK, McNicholas CM, Bartoszewski R, et al. Gliomaspecific cation conductance regulates migration and cell cycle progression. J Biol Chem 2012;287:4053-65.

22. Kapoor N, Bartoszewski R, Qadri YJ, et al. Knockdown of ASIC1 and epithelial sodium channel subunits inhibits glioblastoma whole cell current and cell migration. J Biol Chem 2009;284:24526-41.

23. Jin C, Ye QH, Yuan FL, et al. Involvement of acid-sensing ion channel 1alpha in hepatic carcinoma cell migration and invasion. Tumour Biol 2015;36:4309-17.

24. Gupta SC, Singh R, Asters M, et al. Regulation of breast tumorigenesis through acid sensors. Oncogene 2016;35:4102-11.

25. Edge SB, Compton CC. The American Joint Committee on Cancer: the 7 th edition of the AJCC cancer staging manual and the future of TNM. Ann Surg Oncol 2010;17:1471-4.

26. Joeres N, Augustinowski K, Neuhof A, et al. Functional and pharmacological characterization of two different

Cite this article as: Yang C, Zhu Z, Ouyang X, Yu R, Wang J, Ding G, Jiang F. Overexpression of acid-sensing ion channel 1a (ASIC1a) promotes breast cancer cell proliferation, migration and invasion. Transl Cancer Res 2020;9(12):7519-7530. doi: $10.21037 /$ tcr-20-2115
ASIC1a/2a heteromers reveals their sensitivity to the spider toxin PcTx1. Sci Rep 2016;6:27647.

27. Berdiev BK, Xia J, Jovov B, et al. Protein kinase C isoform antagonism controls BNaC2 (ASIC1) function. J Biol Chem 2002;277:45734-40.

28. Leonard AS, Yermolaieva O, Hruska-Hageman A, et al. cAMP-dependent protein kinase phosphorylation of the acid-sensing ion channel-1 regulates its binding to the protein interacting with C-kinase-1. Proc Natl Acad Sci U S A 2003;100:2029-34.

29. Thrasivoulou C, Millar M, Ahmed A. Activation of intracellular calcium by multiple Wnt ligands and translocation of beta-catenin into the nucleus: a convergent model of $\mathrm{Wnt} / \mathrm{Ca} 2+$ and $\mathrm{Wnt} /$ beta-catenin pathways. J Biol Chem 2013;288:35651-9.

30. Jin C, Yuan FL, Gu YL, et al. Over-expression of ASIC1a promotes proliferation via activation of the beta-catenin/ LEF-TCF axis and is associated with disease outcome in liver cancer. Oncotarget 2017;8:25977-88.

31. Chaturvedi MM, Sung B, Yadav VR, et al. NF-kappaB addiction and its role in cancer: 'one size does not fit all'. Oncogene 2011;30:1615-30.

32. Gupta SC, Singh R, Pochampally R, et al. Acidosis promotes invasiveness of breast cancer cells through ROSAKT-NF-kappaB pathway. Oncotarget 2014;5:12070-82. 